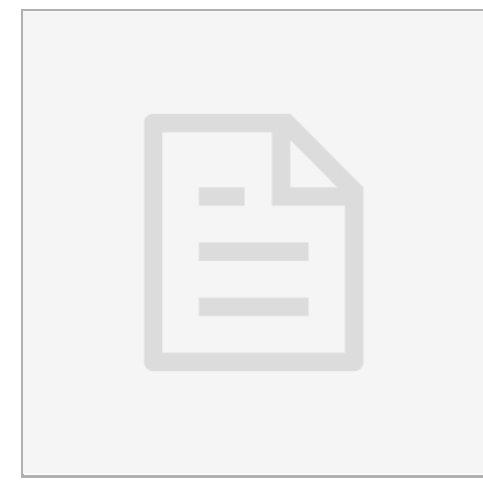

OCT 14, 2019

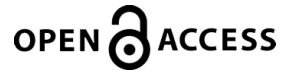

DOI:

dx.doi.org/10.17504/protocol s.io.6j4hcqw

Protocol Citation: Elinor Gottschalk, Bulent Arman Aksoy, Pinar Aksoy, Jeff Hammerbacher 2019. In vitro pmel-1 T cell-mediated cytotoxicity assay with CytoTox-ONE Homogenous Membrane Integrity Assay (Promega). protocols.io https://dx.doi.org/10.17504/p rotocols.io.6j4hcqw

License: This is an open access protocol distributed under the terms of the Creative Commons Attribution License, which permits unrestricted use, distribution, and reproduction in any medium, provided the original author and source are credited

Protocol status: Working We use this protocol and it's working

Created: Aug 15, 2019

Last Modified: Oct 14, 2019

PROTOCOL integer ID:

26972

\section{(3) In vitro pmel-1 T cell-mediated cytotoxicity assay with CytoTox-ONE Homogenous Membrane Integrity Assay (Promega)}

\author{
Elinor Gottschalk ${ }^{1}, \quad$ Bulent Arman Aksoy ${ }^{1}, \quad$ Pinar Aksoy ${ }^{1}$, \\ Jeff Hammerbacher ${ }^{1}$ \\ ${ }^{1}$ Medical University of South Carolina
}

Hammer Lab

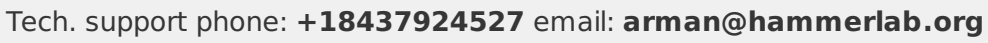

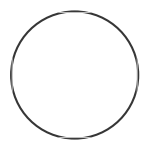

\section{Elinor Gottschalk}

\section{ABSTRACT}

A plate-based assay to estimate the cytotoxic activity of pmel-1 T cells against target cancer cells. The CytoTox-ONE Homogeneous Membrane Integrity Assay from

Promega (Madison, WI) is a lactate dehydrogenase (LDH) release-based assay. Dying cells have damaged cell membranes causing cytosolic components, such as $\mathrm{LDH}$, to leak into the culture media. The CytoTox-ONE assay uses LDH in the culture media to convert resazurin to fluorescent resofurin. The resulting fluorescent signal is proportional to the number of non-viable cells in a sample. One advantage to using this assay for T cell-mediated cytotoxicity is that the cancer cells do not need to be removed from the wells to measure cell death, which is especially convenient with adherent cancer cell types that would require trypsinization. However, we have found that the assay produces noisy data and a set of control wells with $T$ cells alone is required to subtract background, overall increasing the number of replicates that are necessary. We had consistent results when culturing the pmel-1 T cells with hgp100 peptide-pulsed MC38 colon cancer cells. But we had inconsistent results using pmel-1 T cells and their natural target, B16-F10 melanoma cells. This setup could be adapted for $T$ cells other than pmel- 1 and their target cells. The ratio of $T$ cells to cancer cells and the duration of co-culture would have to be optimized. 


\section{MATERIALS}

\section{MATERIALS}

89 CytoTox-ONE(TM) Homogen Membrn Integrity Assay, 200-800 assay Promega Catalog \#G7890

88 CytoOne T75 filter cap TC flask USA Scientific Catalog \#CC7682-4875

$\$ 8$ CytoOne 96-well TC plate USA Scientific Catalog \#CC7682-7596

88 hgp100(25-33) Genscript Catalog \#RP20344

88 B16-F10 cell line (ATCC $\AA$ CRL-6475 ${ }^{\text {TM }}$ ) ATCC Catalog \#CRL-6475

88 MC38 cell line Contributed by users Catalog \#ENH204-FP

STEP MATERIALS

88 CytoTox-ONE(TM) Homogen Membrn Integrity Assay, 200-800 assay Promega Catalog \#G7890

\section{BEFORE START INSTRUCTIONS}

Start culturing T cells:

1. Day 0: Thaw frozen splenocytes or isolate fresh splenocytes

2. Supplement $T$ cell culture media with 200 IU IL-2.

3. Activate the cells.

4. Day $3-6$ : Maintain the T cells at 1 million cells $/ \mathrm{ml}$, splitting when needed. Replenish IL-2 each day at $200 \mathrm{IU} / \mathrm{ml}$.

1 The day before adding T cells to co-culture with the target cancer cells, plate the target cells in a 96 well plate at 25,000 cells per well in DMEM with $10 \%$ FBS.

An example plate layout is below:

- Plate the target cells in columns 2-7. Leave the rest of the wells empty.

- Each column has 6 replicates (we do not recommend reducing the number of replicates because the resulting data is too noisy)

- One plate per condition being tested is required (in this case, the minimum will be two plates, one will have the target cells pulsed with the peptide, one with unpulsed cells as a negative control).

Sample plate layout: 


\begin{tabular}{|c|c|c|c|c|c|c|c|c|c|c|c|c|}
\hline & 1 & 2 & 3 & 4 & 5 & 6 & 7 & 8 & 9 & 10 & 11 & 12 \\
\hline a & & & & & & & & & & & & \\
\hline b & & \multirow{6}{*}{$\stackrel{\ddot{\theta}}{\ddot{\theta}}$} & \multirow{6}{*}{$\stackrel{\vec{\sim}}{\mathrm{i}}$} & \multirow{6}{*}{$\stackrel{\overbrace{}}{ت}$} & \multirow{6}{*}{$\stackrel{\sim}{\sim}$} & \multirow{6}{*}{ 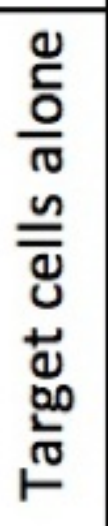 } & \multirow{6}{*}{ 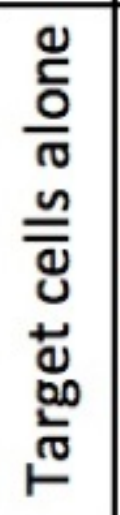 } & \multirow{6}{*}{ 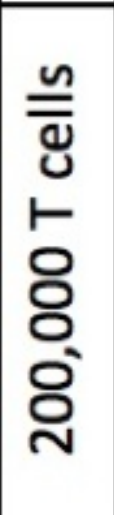 } & \multirow{6}{*}{ 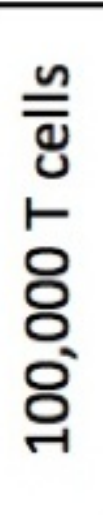 } & \multirow{6}{*}{ 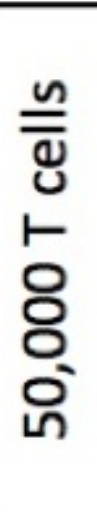 } & \multirow{6}{*}{ 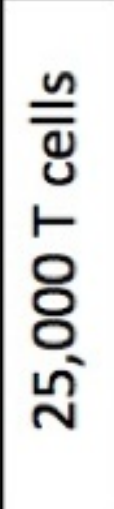 } & \multirow{6}{*}{ 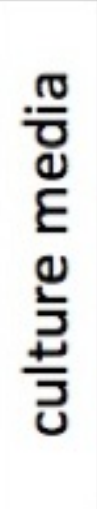 } \\
\hline C & & & & & & & & & & & & \\
\hline d & & & & & & & & & & & & \\
\hline e & & & & & & & & & & & & \\
\hline$T$ & & & & & & & & & & & & \\
\hline g & & & & & & & & & & & & \\
\hline$h$ & & & & & & & & & & & & \\
\hline
\end{tabular}

Incubate the target cells overnight to allow them to adhere to the plate.

2 Prepare the target cells for co-culture:

1. If required, pulse the target cells in one plate with $1 \mathrm{uM} \mathrm{hgp100}$ (peptide for $\circlearrowleft$ 01:00:00 at

\section{$8^{\circ} 37^{\circ} \mathrm{C}$}

2. Wash the peptide out $3 x$ with $T$ cell media

Prepare T cells for co-culture (the day post-activation will depend on your experiments).

1. Centrifuge the T cells at $\because 350 \mathrm{xg}$ for $00: 05: 00$ and resuspend in T cell media supplemented with IL-2 (200IU/ml) to obtain a concentration of 2 million cells per $\mathrm{ml}$

Co-culture T cells with target cells:

1. Aspirate culture media from 96 well plate

2. Pipette $\triangle 100 \mu \mathrm{L}$ T cell media into columns 3-7 and 9-12 (leaving columns 2 and 8 empty)

3. Pipette \& $100 \mu \mathrm{L}$ of $\mathrm{T}$ cell suspension into columns $2,3,8$ and 9

4. Serial dilute the T cells in the plate: there will now be 200 ul total volume in columns 3 and 9 . Starting from column 3 , pipette up and down 3 times to mix. Then transfer $\Delta 100 \mu \mathrm{L}$ to column 4. Pipette up and down to mix in column 4 and transfer $\Omega 100 \mu \mathrm{L}$ to column 5. Pipette up and down to mix in column 5 and remove $\triangle 100 \mu \mathrm{L}$ and discard. Repeat starting at column 9 to make the T cell dilutions without cancer cells.

5. Incubate plates for $24: 00: 00$ at $8^{\circ} 37^{\circ} \mathrm{C}$

3 Perform CytoTox-ONE Homogenous Membrane Integrity Assay:

1. Thaw assay buffer and stop solution from CytoTox-ONE Homogenous Membrane Integrity Assay kit and bring to room temperature. 
1. Thaw lysis solution and make a 1:5 dilution of lysis solution in PBS - total volume 100 ul per plate; this is good for 1 plate ( 80 ul PBS +20 ul lysis solution)

2. Take plate/s out of incubator and place in hood

3. As soon as taking plates out of the incubator add $\triangle 10 \mu \mathrm{L}$ diluted lysis solution to each well in column 7 to lyse the cells for the maximum LDH release control. Make sure to mix the lysis buffer into the well by pipetting up down.

4. Allow plates to sit in hood for $00: 30: 00$ to equilibrate to room temperature

5. Add $11 \mathrm{ml}$ Assay Buffer into one substrate bottle and mix

6. Pour the assay buffer/substrate mixture into a reagent reservoir and using multichannel pipette, pipette $\triangle 100 \mu \mathrm{L}$ into each well to assay in the plate, shake plate gently to mix

7. Cover from light in hood and incubate for $00: 10: 00$

8. Add I $50 \mu \mathrm{L}$ stop solution to each well in the same order as the assay buffer was added, shake plate gently to mix

9. Directly read plates on a fluorescence plate reader (excitation 560, emission 590) in the order that they were prepared in

4 Calculate percent cytotoxicity:

((experimental-CMB)-(Tcellbackground-CMB)/(maxLDHrelease-CMB))X 100

where experimental is the co-culture well (columns 2-6), CMB (culture medium background) is culture media with no cells (column 12), $T$ cell background is $T$ cells only at the different concentrations corresponding to the co-culture ratios (columns 8-11), and max LDH release is MC38 cells lysed with a detergent to release all of the LDH in the cells into the culture media (column 7). 